\title{
Diagnosis of a complex chromosomal rearrangement using fluorescent in situ hybridisation
}

Division of Medical Genetics, Jefferson Medical College of Thomas Jefferson University, 1100 Walnut Street, Suite 400, Philadelphia, PA 19107-5563, USA $\mathrm{R}$ Wallerstein L Gibas

$\mathrm{C}$ E Anderson

L Jackson

Correspondence to: Dr Wallerstein.

Received 7 August 1995 Revised version accepted for publication 19 April 1996

\author{
Robert Wallerstein, Longina Gibas, Carol E Anderson, Laird Jackson
}

\begin{abstract}
We report the use of fluorescent in situ hybridisation (FISH) to clarify a complex chromosomal rearrangement (CCR) carried by a woman presenting with recurrent miscarriages. CCRs are rare cytogenetic rearrangements involving three or more chromosomes, which can be difficult to interpret using routine cytogenetic studies with GTG banding. FISH was used to establish a correct interpretation of the maternal karyotype before amniocentesis in a present pregnancy.

( $¥$ Med Genet 1996;33:793-794)
\end{abstract}

Key words: complex chromosomal rearrangements (CCR); fluorescent in situ hybridisation (FISH); prenatal diagnosis; recurrent spontaneous abortion. (CCR) involve rearrangement of chromosomal material between three or more chromosomes with three or more breakpoints, which present difficulty in interpretation. ${ }^{12}$ The rearrangements can include deletions, insertions, or inversions that distort GTG banding patterns. ${ }^{3}$ Fluorescent in situ hybridisation (FISH) has been used to identify the origin of rearranged chromosomal material. ${ }^{3-5}$ We report a case of a CCR carrier identified through recurrent spontaneous abortion in whom FISH studies were vital for correct interpretation of an unbalanced fetal chromosome karyotype in a continuing pregnancy.
Complex chromosomal rearrangements

\section{Case report}

The proband's obstetric history included three spontaneous abortions at 12,14 , and 20 weeks, as well as delivery of a healthy son in her third shown an abnormal fetal karyotype interpreted by GTG banding as $46, \mathrm{XX},-5,-$ $7,+\operatorname{der}(5) \mathrm{t}(5 ; 7)(\mathrm{p} 15 ; \mathrm{q} 31),+\operatorname{der}(7) \mathrm{t}(7 ; 11) \quad(\mathrm{q} 31 ;$ q13). When she presented for prenatal diagnosis at 16 weeks' gestation in her fifth pregnancy, she was identified as a carrier of a CCR involving chromosomes 5,7 , and 11 (fig 1). Her par-

In order to perform prenatal diagnosis, FISH was performed using a maternal blood sample in separate hybridisations using two probes from Oncor, Inc, Gaithersburg, MD: cri du chat cosmid probe with assignment at 5 p15.3 (fig 2A) and chromosome 7 specific painting probe (fig $2 \mathrm{~B}$ ). The study showed a small chromosome segment containing 5p15.3 inserted into the $\mathrm{q}$ arm of a submetacentric chromosome identified on GTG banding as a derivative 7 . Inversion of the inserted segment is possible, because its orientation could not be determined. FISH using the 7 painting probe showed chromosome 7 derived material on a large submetacentric chromosome identified as a derivative 5 . The 7 paint probe did not hybridise evenly, but was satisfactory for identification of the rearranged material on the derivative chromosomes. A chromosome 11 paint probe was not necessary as the 11 material was clearly identifiable on routine banding. Combining the information from GTG bandpregnancy. Tissue from her 20 week loss had ents and her husband had normal karyotypes.
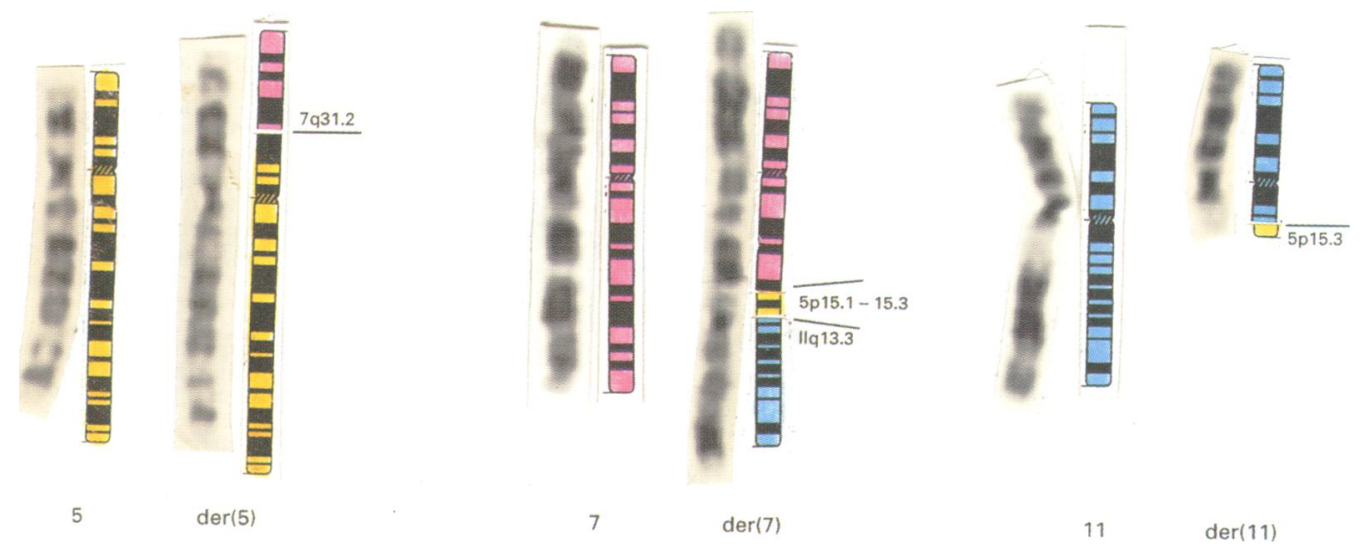

Figure 1 Partial ideogram and GTG banded karyotype of the patient showing normal chromosomes 5, 7, 11, and their derivatives. Ideogram shows rearrangements as interpreted after FISH studies. 

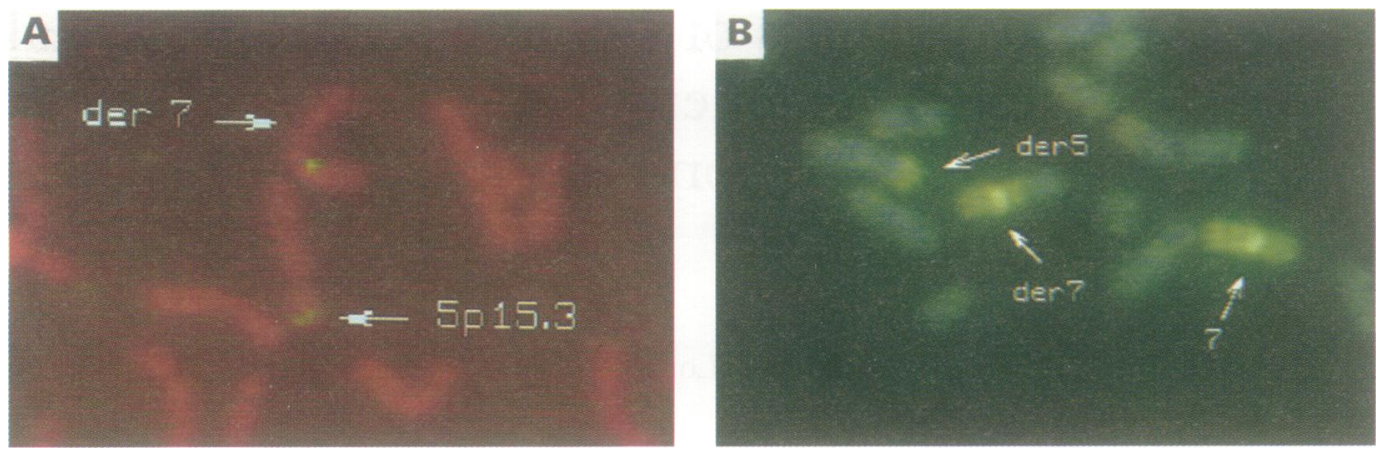

Figure 2 Partial view of metaphase from lymphocyte culture showing FISH study: $(A)$ cri du chat cosmid probe indicating 5p15.3 inserted into derivative chromosome 7 and normal homologue, (B) painting probe for chromosome 7 showing translocated 7 material on derivative 5, derivative 7 , and normal homologue.

ing and FISH analysis, the breakpoints of the three way translocation were established as 5p15.1, 5p15.3, 7q31.2, and 11q13.3. The final interpretation of the balanced maternal karyotype was $46, \mathrm{XX}, \mathrm{t}(5 ; 7 ; 11)(5 \mathrm{qter} \rightarrow$ 5p15.1::7q31.2 $\rightarrow$ 7qter;7pter $\rightarrow 7 q 31.2:: 5$ p 15.3 5 p15.1::11q13.3 $\rightarrow$ 11qter;11pter $\rightarrow 11$ q13.3:: 5 p $15.3 \rightarrow 5$ pter $)$

Ultrasound studies at 17 weeks, when amniocentesis was performed, did not detect any fetal abnormalities. Cytogenetic analysis of fetal cells showed an unbalanced karyotype trisomic for 7q31.2 to 7qter and monosomic for $5 \mathrm{p} 15.1$ to 5pter: 46,XY,$5,+\operatorname{der}(5) \mathrm{t}(5 ; 7 ; 11)$ mat. Since the derivative chromosome 5 was identified through FISH studies of the maternal karyotype, GTG studies were sufficient for prenatal diagnosis.

Duplication of $7 q$ in the region of $7 q 3$ has resulted in pre- and postnatal growth retardation, severe to profound mental retardation, hypotonia, seizures, hydrocephalus, and neonatal death. ${ }^{6}$ Deletion of $5 \mathrm{p}$ results in the cri du chat syndrome, associated with severe mental retardation, microcephaly, growth retardation, epicanthic folds, and a monochromatic cry similar to the mewing of a cat. ${ }^{7}$ Because of the severity of the prognosis, the patient decided to terminate the affected pregnancy.

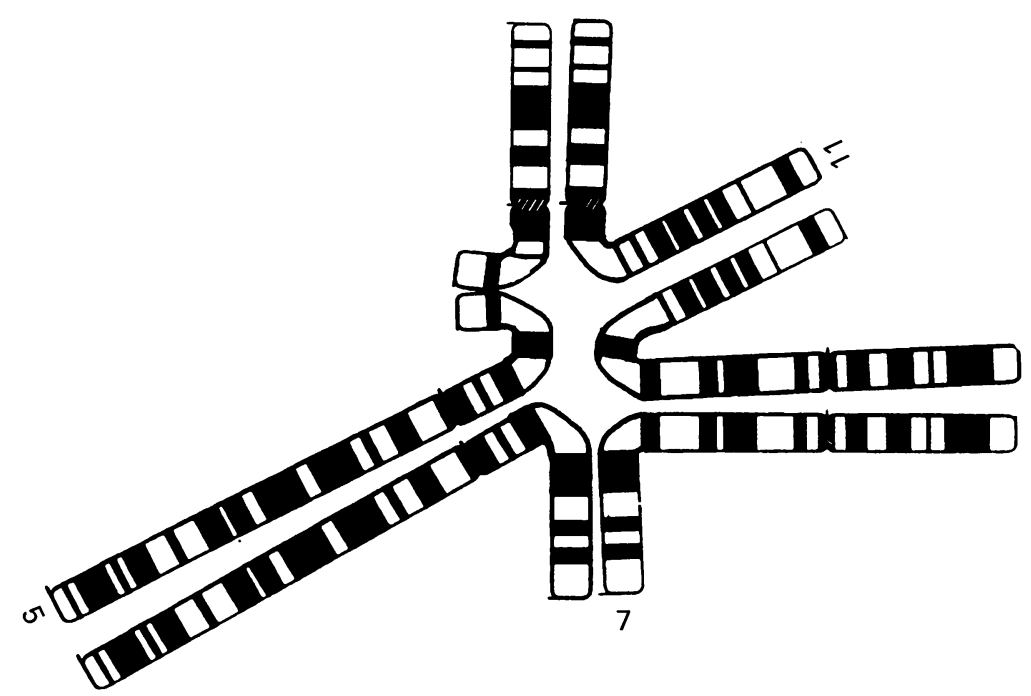

Figure 3 Theoretical multivalent chromosome pairing in pachytene of meiosis 1 showing the intricacy involved in chromosome pairing and assortment with the 5, 7,11 translocation.

\section{Discussion and conclusions}

Most carriers of CCRs are ascertained through recurrent miscarriages or the birth of an abnormal child. ${ }^{8}$ Recurrence risks for pregnancy loss are empirically estimated to be as high as $48 \%$, and the risk for birth of an abnormal child may be as high as $18 \% .^{9}$ Fig 3 shows the theoretical meiotic pairing of the rearranged chromosomes to illustrate the intricate segregation needed to produce a balanced gamete in this case.

In previously reported CCR cases, when FISH was used to supplement GTG studies, the rearrangements were correctly identified to be more complex. ${ }^{31011}$ In the reported case, FISH studies allowed accurate prenatal diagnosis of complex cytogenetic findings and more specific prediction of potential fetal abnormalities.

We are grateful to Dr Susan Cowchock for editorial assistance with the manuscript.

1 Kleczkowska A, Fryns JP, Van Den Berghe. Complex chromosomal rearrangements (CCR) and their genetic consequences. Am f Hum Genet 1982;30:199-214.

2 Wang H, McLaughlin M, Thompson C, Hunter AGW. Use of fluorescence in situ hybridization to confirm the of fluorescence in situ hybridization to confirm the interpretation of a balanced complex chromosomal rearrangement ascertained thro

3 Batista DAS, Tuck-Miller CM, Martinez JE, et al. A complex chromosomal rearrangement detected prenatally and studied by fluorescence in situ hybridization. Hum Genet 1993;62:1177-21

4 Batista DAS, Pai S, Stetten G. Molecular analysis of a complex chromosomal rearrangements by in situ hybridization. Am f Med Genet 1994;53:255-63.

5 Rosenberg C, Blakemore KJ, Kearns WG, et al. Detection of chromosomal rearrangements by in situ hybridization. $A m$ 7 Hum Genet 1992;50:700-5.

6 Schinzel AA. Chromosome 7, trisomy 7q2-3. In: Buyse MI ed. Birth defects encyclopedia. Cambridge, Mass: Blackwel Scientific Publications, 1990:349-50.

7 Niebuhr E. The cri du chat syndrome: epidemiology, cytogenetic, and clinical features. Hum Genet 1978 cytogenetic,

8 Saadallah N, Hulten M. A complex three breakpoint translocation involving chromosomes 2,4 , and 9 identified by location involving chromosomes 2, 4, and 9 identified by meiotic investigations of a human ma

9 Kausch $\mathrm{K}$, Haaf $\mathrm{T}$, Kohler $\mathrm{H}$, Schmid M. Complex Kausch $\mathrm{K}$, Haaf $\mathrm{T}$, Kohler $\mathrm{H}$, Schmid $\mathrm{M}$. Complex
chromosomal rearrangement in a woman with multiple

10 Gorski JL, Kistenmacher ML, Punnett HH, et al. Reproductive risks for carriers of complex chromosomal rearrangements: analysis of 25 families. Am $\mathcal{f}$ Med Genet 1988;29:247-61

1 Kuwano A, Ledbetter SA, Dobyns WB, et al. Detection of deletions and cryptic translocations in Miller-Dieker syndrome by in situ hybridization. Am f Hum Genet 1991; 49:707-14. 